\title{
Curcumin induced autophagy anticancer effects on human lung adenocarcinoma cell line A549
}

\author{
FURONG LIU $^{1}$, SONG GAO ${ }^{1}$, YUXUAN YANG ${ }^{1}$, XIAODAN ZHAO $^{1}$, YAMENG FAN ${ }^{1}$, \\ WENXIA MA ${ }^{1}$, DANRONG YANG ${ }^{1}$, AIMIN YANG ${ }^{2}$ and YAN YU ${ }^{1}$ \\ ${ }^{1}$ Department of Public Health, Medical College of Xi'an Jiaotong University; ${ }^{2}$ Department of \\ Nuclear Medicine, The First Affiliated Hospital of Xi'an Jiaotong University, Xi'an, Shaanxi 710061, P.R. China
}

Received February 16, 2017; Accepted June 28, 2017

DOI: $10.3892 / \mathrm{ol} .2017 .6565$

\begin{abstract}
To investigate the anticancer effects of curcumin-induced autophagy and its effects on the human lung adenocarcinoma A549 cell line, inverted phase contrast microscopy was used to observe alterations to the cytomorphology of cells. An MTT assay was used to measure cell viability. Autophagy was detected using acridine orange (AO) staining and 3-methyladenine (3-MA) was used as an autophagy-specific inhibitor. Dose- and time-dependent A549 cell viability inhibition was observed following curcumin treatment. A dose-dependent increase in the red fluorescent structures in A549 cells was identified following curcumin treatment for $48 \mathrm{~h}$ through $\mathrm{AO}$ staining. In addition, the activation of autophagy was determined through changes in the number of autophagic vesicles (AVs; fluorescent particles) infected with monodansylcadaverine (MDC). The fluorescence intensity and density of AVs in the curcumin-treated groups were higher at $48 \mathrm{~h}$ compared with the control group. Finally, the MTT assay demonstrated that the survival rates of the curcumin-treated cells were increased when pretreated with 3-MA for $3 \mathrm{~h}$, indicating that the inhibitory effect of curcumin on A549 cells is reduced following the inhibition of autophagy. Furthermore, AO and MDC staining confirmed that 3-MA does inhibit the induction of autophagy. Thus, it was hypothesized that the induction of autophagy is partially involved in the reduction of cell viability observed following curcumin treatment. The anticancer effects of curcumin on A549 cells can be reduced using autophagy inhibitors. This suggests a possible cancer therapeutic application of curcumin through the activation of autophagy. These findings have
\end{abstract}

Correspondence to: Professor Aimin Yang, Department of Nuclear Medicine, The First Affiliated Hospital of Xi'an Jiaotong University, 277 West Yanta Road, Xi'an, Shaanxi 710061, P.R. China E-mail: yangaimin@mail.xjtu.edu.cn

Professor Yan Yu, Department of Public Health, Medical College of Xi'an Jiaotong University, 76 West Yanta Road, Xi'an, Shaanxi 710061, P.R. China

E-mail:yuyan@mail.xjtu.edu.cn

Key words: curcumin, A549 cell, autophagy, 3-MA, anticancer improved the understanding of the mechanism underlying the anticancer property of curcumin.

\section{Introduction}

At present, cancer threatening human health and life is a major public problem and the lung caner is the most prevalent malignant tumor. According to histological classification, lung cancer was divided into non-small cell lung cancer (NSCLC) and SCLC. The former captures more than $80 \%$ and the survival rate is very low due to resistance of adjuvant chemotherapy (1). For many years, medical staffs have been strived their best to find more effective and low cytotoxcity chemotheraputics, especially the natural drugs.

Nature is rich, having provided us with a large number of compounds derived from plants with tumor treatment efficacy caused the attention of the world, because of their availability and relatively low toxicity compared with chemotherapy. Curcumin (diferuloylmethane)-(1,7-bis (4-hydroxy-3-methoxyphenyl)-1,6-hepadiene-3,5-dione), a kind of liposoluble polyphenol pigment extracted from rhizome of curcuma, which has a long history as a dietary supplement is konwn with a variety of biological activity such as anti-inflammatory, anticoagulant, hypolipidemic, antioxidant, free radical scavenging, anti-atherosclerosis, etc. It is the main efficacy components of Chinese medicine turmeric playing a pharmacological effect (2). Recently, clinical trials have shown that curcumin is safe and well tolerated in humans (3). In addition, in vitro, curcumin suppresses the growth of multiple cancer lines, especially in lung cancer (4-6). These findings highly indicating its potential clinic application speculation in cancer control.

Curcumin has been suggested to induce cell cycle arrest and activate apoptosis-mediated cell death in several cancer cells, including lung cancer (7-9). Although it is strongly believed that apoptosis is the main toxic mechanism of curcumin in tumor cells, it is not completely known whether autophagy is induced in curcumin-caused cancer cell death. Simultaneously, in recent years, the fact that curcumin can upregulate autophagy to achieve its antitumor function arouse strong interest.

As one of the three types of programmed cell death (PCD), autophagy, a catabolic process for the degradation 
and recycling of macromolecules and organelles which can be activated during stress conditions was identified as an important point at the tumor control procedure. It can be divided into macrophages, micro-autophagy and molecular chaperone mediated autophagy. It was found that curcumin induced autophagy by downregulating Akt/mTOR and activating ERK1/2 pathway, and this effect was also mediated by AMPK and related to the extensive degradation of P53 (10-13). Autophagy is closely related to tumorigenesis affecting the proliferation, migration, invasion and metastasis of tumor cells. In recent years, an increasing number of research evidence that autophagy may play a dual role in tumors (14). On the one hand, autophagic degradation of amino acids, nucleotides and free fatty acids promote cell survival. On the other hand, it is also an important mechanism of tumor cell death. Autophagy has a dual role in cancer cells, including curcumin-induced autophagy of tumor cells, likely this could be a survival or cell death mechanism. Although the dual role of autophagy has not yet reached a consensus, the role in tumor development and treatment can't be ignored. So, exploring the role of autophagy in tumorigenesis and development and the mechanism may be able to reveal a new chapter in antitumor experience.

In order to investigate the curcumin induced autophagy anticancer effects and the possible mechanism, a variety of methods were used to observe the cytomorphology alteration, viability, curcumin-induced autophagy and autophagy-specific inhibition in A549 cell line. We prospect the results providing a baseline information for better understanding the curcumin induced anticancer mechanism.

\section{Materials and methods}

Materials. The A549 human lung adenocarcinoma cell line were obtained from the First Affiliated Hospital of Xi'an Jiaotong University (Xi'an, China) as a gift.

Curcumin was obtained from Sigma (St. Louis, MO, USA) and dissolved into dimethyl sulphoxide (DMSO) stock solution, kept in the dark, waiting to be used. RPMI-1640 (HyClone, Logan, UT, USA). The MTT, DMSO, 3-methyladenine (3-MA), acridine orange (AO) solution and monodansylcadaverine (MDC) are all obtained from Sigma. Fetal bovine serum (FBS; Biological Industries, Kibbutz Beit-Haemek, Israel). Penicillin-streptomycin (North China Pharmaceutical Group Corp., Shijiazhuang, China). Anti-quencher reagent (Hurt Biotechnology companies, Xi'an, China). The other commonly used reagents are domestic analytical reagents. 3-MA dissolved in PBS was added to the medium to final concentrations as described in each experiment. DMSO is a control for the entire study at a final concentration of $<0.1 \%$. Cells were pretreated with 3-MA of $2.5 \mathrm{mM}$ for $3 \mathrm{~h}$ and then incubated with curcumin for scheduled time of experiment.

\section{Methods}

Cell culture. A549 cells maintained at $37^{\circ} \mathrm{C}, 5 \% \mathrm{CO}_{2}$ and saturated humidity in RPMI-1640 culture medium supplemented with $10 \%$ FBS and $1 \%$ penicillin-streptomycin. Changing the liquid every day, and cells were passaged once every 2 to 3 days, taking the logarithmic growth phase of the A549 cells for following tests.
Morphological observation. $4 \times 10^{4} / \mathrm{ml}$ suspension of A549 cells were seeded in 24-well plates at $1 \mathrm{ml}$ per well cultured overnight until the cells adhered to the wall. The cells were exposed to curcumin $(40 \mu \mathrm{M})$, and the same volume of the consumption of DMSO for solubling curcumin was considered a control group. After cultured for 24, 48, 72 and 96 h, A549 cell morphological changes were observed and photographed under inverted phase contrast microscope (Nikon Eclipse Ti; Nikon Corporation, Tokyo, Japan).

MTT cell viability assay. Collecting the logarithmic growth phase A549 cells seeded at $2 \times 10^{4}$ cells per well in 96-well culture plates and at least 3 wells were replated in each group. Each 96-well plate was set up with control (cells-only) and zero-adjustment (medium only). The following day, the medium was changed to RPMI-1640 supplemented with $10 \%$ fetal bovine serum containing different concentrations of curcumin or DMSO alone (control group). At 24, 48, 72 and $96 \mathrm{~h}$ later, the medium was removed and the A549 cells were incubated with $20 \mu \mathrm{l} \mathrm{MTT}(5 \mathrm{mg} / \mathrm{ml})$ at $37^{\circ} \mathrm{C}$ for $4 \mathrm{~h}$. Carefully aspirating the liquid, adding $150 \mu \mathrm{l}$ DMSO per well. Shaking 5 min and the absorbance of each well was measured with the microplate reader at $570 \mathrm{~nm}$. Cell viability $(\%)=($ experimental group OD-zero adjustment group OD)/(control group OD-zero adjustment group OD) x 100\%. Cell inhibitory ratio (\%)=1-cell viability (\%).

AO staining. During authophagy, autophagosomes fuse with lysosomes to form autophago-lysosomes which could be dyed by AO. Collecting the logarithmic growth phase A549 cells seeded at $3 \times 10^{4}$ cells per well in 24 -well culture plates. Exposed to different concentrations of curcumin after $48 \mathrm{~h}$, cells were stained with $10 \mu \mathrm{g} / \mathrm{ml}$ AO for $15 \mathrm{~min}$. Following treatment, washed twice with PBS, adding $30 \mu \mathrm{l}$ anti-quencher per well and immediately observed under an inverted fluorescence microscope (Nikon Eclipse Ti; Nikon Corporation). The experiment was repeated three times.

Monodansylcadaverine $(M D C)$ labeling. Usually, mature autophagic vacuoles which accumulate in the autophagy are detected by MDC. The same as the AO staining experiment, A549 cells were seeded into 24-well culture plates and treated with 0 (DMSO), 10, 20 and $40 \mu \mathrm{M}$ curcumin, 3-MA, 3 -MA+curcumin $(40 \mu \mathrm{M})$, respectively. At $48 \mathrm{~h}$ latter, the cells were incubated with fresh medium containing MDC $(50 \mu \mathrm{M})$ for $15 \mathrm{~min}$ at $37^{\circ} \mathrm{C}$ and $5 \% \mathrm{CO}_{2}$. The anti-quencher was added after washing twice with PBS and taking pictures with UV excitation by an inverted fluorescence microscope (Nikon Eclipse Ti; Nikon Corporation) quickly. The experiment was repeated three times.

Transmission electron microscopy (TEM). A549 cells treated with $20 \mu \mathrm{M}$ curcumin for $48 \mathrm{~h}$ were rinsed with PBS twice. Firstly, the cells were fixed with $2.5 \%$ glutaraldehyde at $4{ }^{\circ} \mathrm{C}$ for $2 \mathrm{~h}$ and washed with $0.1 \mathrm{M}$ phosphate buffer for $30 \mathrm{~min}$ or more. Then post-fixed in $0.1 \mathrm{M}$ sodium phosphate buffer containing $1 \%$ osmium tetroxide for $2 \mathrm{~h}$ at $4^{\circ} \mathrm{C}$. After a series of ethanol dehydration, embedding, polymerization, slicing and dyeing were carried out. Finally, the thin sections of 50-70 nm stained for $15 \mathrm{~min}$ with uranyl acetate and citric 


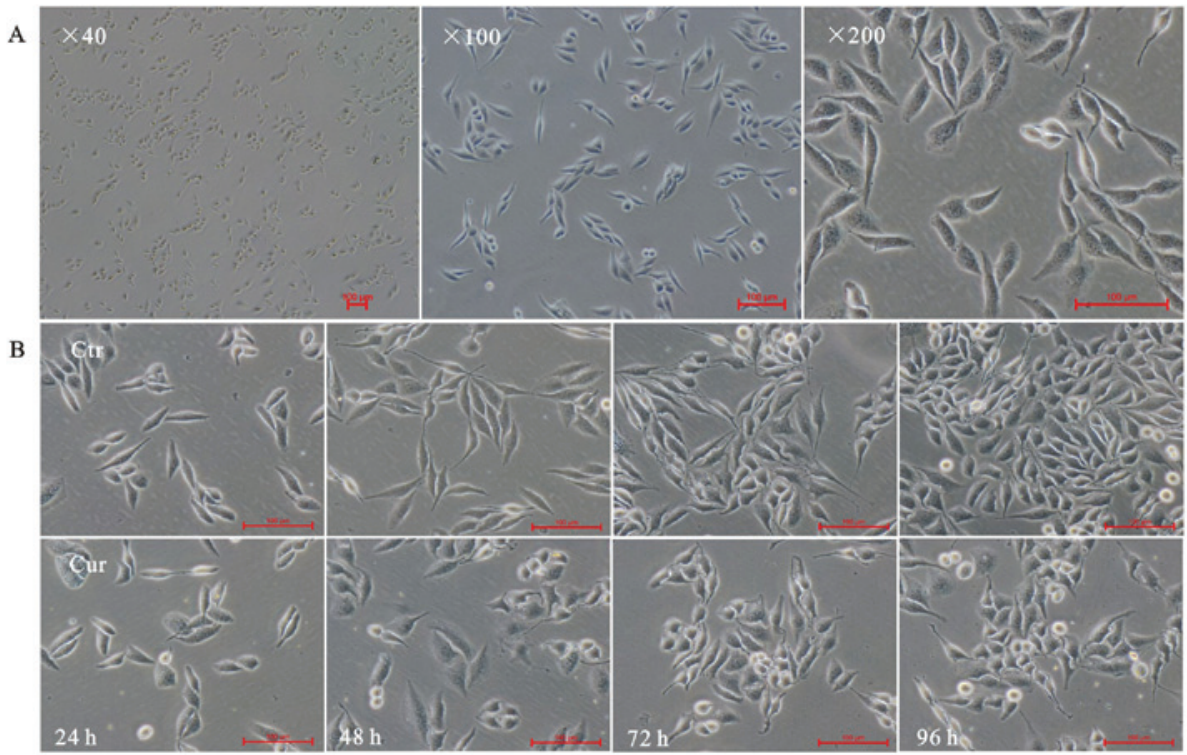

Figure 1. Morphology of lung adenocarcinoma A549 cells under inverted phase contrast microscopy. (A) Morphology of normal A549 cells at different magnification (magnification, $\mathrm{x} 40, \mathrm{x} 100$ and $\mathrm{x} 200$ ). (B) Morphologically, curcumin (Cur; $40 \mu \mathrm{M}$ ) administration induced cell rounding and growth inhibition, which appeared to be time dependent. Control (Ctr) was administered DMSO alone (magnification, x200).

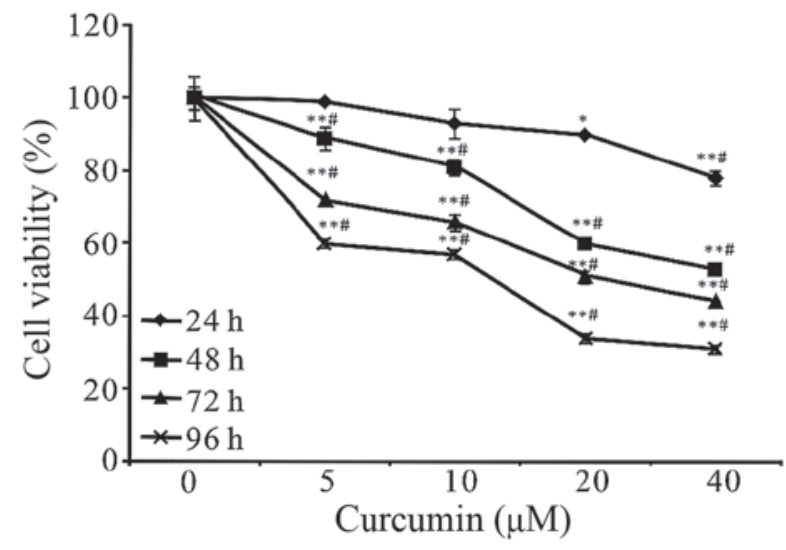

Figure 2. A549 cells were treated with curcumin at the indicated concentrations and time periods. Control is concentration $0 \mu \mathrm{M}$ of curcumin. Cell proliferation was performed using MTT and measuring absorbance at 24, 48, 72 and $96 \mathrm{~h}$. It was shown that curcumin was able to inhibit the growth of A549 cells in a concentration-and time-dependent manner. Data were represented as mean \pm SEM, which are three separate experiments performed in triplicate. $\mathrm{n}=9$ in each group. ${ }^{*} \mathrm{P}<0.05$ and ${ }^{* *} \mathrm{P}<0.01$ for $0 \mu \mathrm{M}$ vs. $5,10,20$ or $40 \mu \mathrm{M} ;{ }^{*} \mathrm{P}<0.05$ for $24 \mathrm{~h}$ vs. 48,72 or $96 \mathrm{~h}$. One-way ANOVA followed by Dunnett's multiple comparision t-test. 3-MA 3-methyladenine.

acid, respectively. Images were obtained by electron microscopy (JEM-1200EX; JEOL, Tokyo, Japan).

Statistical analysis. Each experiment was conducted at least three times. SPSS 18.0 statistical software (SPSS, Inc., Chicago, IL, USA) was used for data analysis. For the measurement data expressed as mean \pm SEM, with a minimum of three separate experiments for each issue addressed. Data were analyzed by one-way ANOVA or factorization variance and comparisons between groups were done using the Dunnett's or LSD t-test. $\mathrm{P}<0.05$ was considered to indicate a statistically significant difference; and $\mathrm{P}<0.01$ was considered to indicate a highly significant difference.

\section{Results}

Cell morphology. The inverted microscope was used to exam A549 cells morphology alteration. As expected, normal lung adenocarcinoma A549 cells showed a long fusiform shape, small size, clear cell boundaries, well-adherent pebble-like growth, placental cytoplasm and less cytoplasmic granules (Fig. 1A). Cells were treated with $40 \mu \mathrm{M}$ curcumin, and pictures were taken at indicated times (24, 48, 72 and $96 \mathrm{~h})$. The results showed that curcumin could inhibit growth of A549 cells. Curcumin made A549 cells rounding and normal spindle shape disappeared, compared with the corresponding time point of control group (Fig. 1B-Ctr), bright-circular dead cells floating increased (Fig. 1B-Cur).

Curcumin affected the A549 cell viability. Succinate dehydrogenase (SDH) in living cells can reduce the exogenous MTT to insoluble formazan, a blue-violet crystalline, and dead cells without this function. The formazan in a cell can be dissolved in DMSO. Using a microplate reader to measure the absorbance at $570 \mathrm{~nm}$ (OD value) can indirectly reflect the number of living cells. Indirectly, absorbance at $570 \mathrm{~nm}$ (OD value) which reflects the number of living cells, can be measured by microplate reader (Infinire M200; Tecan, Männedorf, Switzerland). The larger the OD value, the more live cells, the stronger of activity.

To assess the effects of curcumin on the cell viability of A549 cells, the cells were treated with curcumin at different concentrations $(0,5,10,20$ and $40 \mu \mathrm{M})$ for different time periods $(24,48,72$ and $96 \mathrm{~h})$ and then followed by MTT assays. It has been observed that the growth inhibition of curcumin on A549 cells was manifested in a concentration-and time-dependent manner (Fig. 2). In addition, we found that the cell viability could be significantly reduced with an incubation period of $48 \mathrm{~h}$ at 20 and $40 \mu \mathrm{M}$. Therefore, the $48 \mathrm{~h}$ time point was used for further studies. Treatment time of curcumin was 
Table I. The results of factorial analysis of curcumin-treated cell viability. Interaction and single-effect significance were found on treatment time and concentration of curcumin.

\begin{tabular}{lcccccccc}
\hline & \multicolumn{7}{c}{ Curcumin $(\mu \mathrm{M})$} \\
\cline { 2 - 7 } Time (h) & Control & 5 & 10 & 20 & 40 & Total & F & P-value \\
\hline 24 & $0.00 \pm 0.01$ & $0.01 \pm 0.01$ & $0.07 \pm 0.04$ & $0.10 \pm 0.01$ & $0.22 \pm 0.02$ & $0.08 \pm 0.02$ & 14.81 & $<0.01$ \\
48 & $0.00 \pm 0.03$ & $0.11 \pm 0.03$ & $0.19 \pm 0.02$ & $0.40 \pm 0.02$ & $0.47 \pm 0.01$ & $0.23 \pm 0.03$ & 62.92 & $<0.01$ \\
72 & $0.00 \pm 0.01$ & $0.28 \pm 0.00$ & $0.34 \pm 0.02$ & $0.49 \pm 0.01$ & $0.56 \pm 0.01$ & $0.34 \pm 0.03$ & 462.78 & $<0.01$ \\
96 & $0.00 \pm 0.06$ & $0.40 \pm 0.01$ & $0.43 \pm 0.01$ & $0.66 \pm 0.01$ & $0.69 \pm 0.01$ & $0.44 \pm 0.04$ & 109.26 & $<0.01$ \\
Total & $0.00 \pm 0.02$ & $0.20 \pm 0.03$ & $0.26 \pm 0.03$ & $0.41 \pm 0.04$ & $0.49 \pm 0.03$ & $0.27 \pm 0.02$ & $236.47^{\mathrm{a}}$ & $<0.01^{\text {a }}$ \\
F & 0.000 & 93.243 & 39.36 & 261.59 & 315.11 & $295.58^{\mathrm{a}}$ & $17.96^{\mathrm{b}}$ & $<0.01^{\mathrm{b}}$ \\
$\mathrm{P}$ & 1.000 & $<0.01$ & $<0.01$ & $<0.01$ & $<0.01$ & $<0.01^{\mathrm{a}}$ & & \\
\hline
\end{tabular}

Data were represented as mean \pm SEM. ${ }^{\mathrm{a}, \mathrm{b}}$ Indicate the F-statistic and P-value of the main effect and interaction effect, respectively.

analyzed by single-effect analysis. As shown in Table I, the cell inhibitory rates were all significantly different $(\mathrm{P}<0.01)$ in the 5, 10, 20 and $40 \mu \mathrm{M}$ groups $(\mathrm{F}=93.243 ; 39.36 ; 261.59$ and 315.11, respectively) with different time intervention. Similarly, there were statistical significance $(\mathrm{P}<0.01)$ in 24,48 , 72 and 96 hroups $(\mathrm{F}=14.81 ; 62.92 ; 462.78$ and 109.26, respectively) when the curcumin concentrations were analyzed by single-effect analysis of the cell inhibitory rates. In short, the results of a single-effect analysis of the time and concentration of curcumin treatments were both statistically significant in cell inhibition, $\mathrm{P}<0.01$. Also, there was an interaction between time and concentration of curcumin treatments, $\mathrm{P}<0.01$, both contributing to the inhibition of proliferation of A549 cells.

Curcumin triggered autophagy in A549 cells. To investigate whether curcumin triggers autophagy in A549 cells, the morphological changes after curcumin treatment were checked by AO, MDC staining and the TEM.

First, monitored the formation of autophagic vesicles (AVs) by AO staining. Autophagosomes fuse with lysosomes forming antophagolysosomes as acidic vesicular organelles (AVOs) can bind AO in the process of cells autophagy (15). AO can stain nuclear and cytoplasm with bright green and make AVOs with bright red. Formating and promoting the AVOs is one of the characters of autophagy (16). The fluorescence microscope was used to measure the changes of AVOs which could be investigated by the appearance of red fluorescence. Our research showed that AVOs could be detected in curcumin-treated A549 cells by AO staining analysis, which suggested that curcumin may induce A549 cells autophagy. In addition, we found that curcumin administration increased the proportion of red-stained AVOs at $48 \mathrm{~h}$ in a dose-dependent manner in the A549 cells by fluorescence microscopy (Fig. 3A). Also, the percentage of curcumin-treated cells with AVOs was counted in the images, which existed increasing in a concentration-dependent manner at $48 \mathrm{~h}$ (Fig. 3B). In addition, the pretreatment with 3-MA for $3 \mathrm{~h}$ attenuated curcumin-induced autophagy in A549 cells according to the impaired red fluorescence (Fig. 3C) which corresponded to the percentage of AVOs in curcumin-treated cells (Fig. 3D).

Toconfirm autophagy induction by curcumin, MDC staining was performed. The activation of autophagy was inferred from changes in fluorescent particles (AVs) infected with MDC. As shown in Fig. 4, compared with curcumin-untreated control, curcumin-treated A549 cells resulted in an increase of fluorescence intensity and density at $48 \mathrm{~h}$ indicating extensive MDC-positive autophagic vacuoles. We found that curcumin administration increased the proportion of MDC-infected AVs at $48 \mathrm{~h}$ in a dose-dependent manner. Moreover, exposed to $40 \mu \mathrm{M}$ curcumin after the pretreatment with 3 -MA for $3 \mathrm{~h}$, MDC fluorescence particles were significantly reduced in A549 cells. As expected that the fluorescence intensity of the 3-MA alone treatment group was the lowest. It was further demonstrated that curcumin treatment induced autophagy which was inhibited by 3-MA in A549 cells.

TEM of autophagosomes is the most direct way to examine autophagy, which is a classical method to check autophagy activation. Furthermore, the number of autophagic vacuoles presenting in A549 cells were investigated using TEM analysis. Images (Fig. 5-Ctr) showed normal cytoplasm, organelles and nucleus morphology of control group, at $48 \mathrm{~h}$ in the cytoplasm. However, curcumin administration $(20 \mu \mathrm{M})$ induced the formation of multiple vacuole-like and double-membrane-enclosed structures were considered to be autophagosomes as shown by the black arrows in the images (Fig. 5-Cur).

All the results above have provided real evidence for curcumin-induced autophagy of A549 cells.

Autophagy inhibitor promoted the curcumin-treated A549 cells survival. As above, curcumin could induced autophagy (Figs. 3A and B, and 4) and significant differences by $\mathrm{AO}$ staining and MDC labeling analysis of treated A549 cells in the presence of 3-MA at $48 \mathrm{~h}$ implied autophagy did work at that time (Figs. 3C and D, and 4). What is the role of autophagy induced by curcumin in inhibiting A549 cell growth? We pretreated the cells with 3-MA for $3 \mathrm{~h}$ to block autophagy following MTT method to detect the proliferation of cells. As shown in Fig 6, the treatment of A549 cells with 2.5 mM 3-MA for $48 \mathrm{~h}$ did not affect cell viability. When 3-MA was pre-added $(3 \mathrm{~h})$ into the curcumin-treated $(10,20$ and $40 \mu \mathrm{M})$ A5 49 cells at $48 \mathrm{~h}$, the cell survival rates both increased significantly at each treatment concentrations of curcumin. These results suggested that autophagy was induced in A549 cells by 


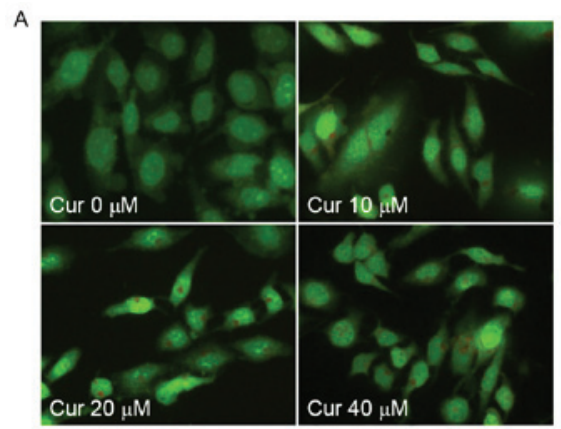

B
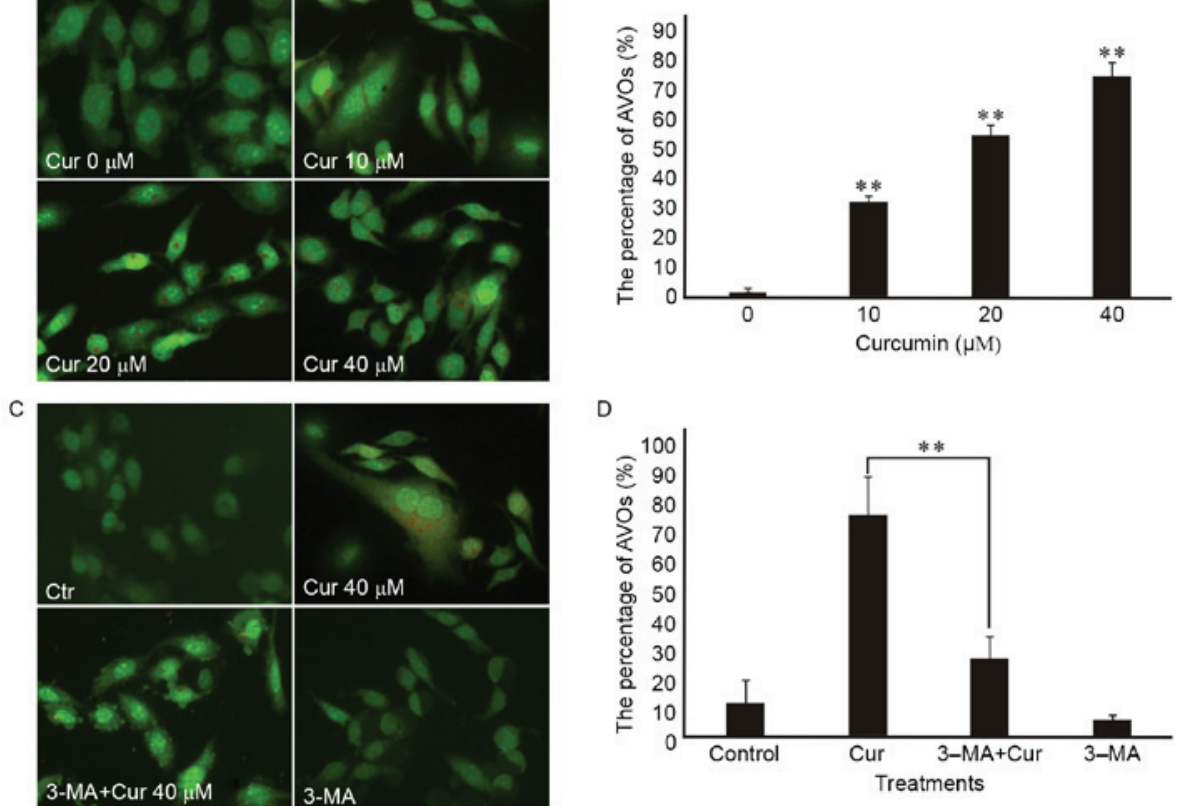

Figure 3. Microscopic detection of AVOs in A549 cells by acridine orange (AO) staining. Control (Ctr) is concentration $0 \mu \mathrm{M}$ of curcumin (Cur). AO can stain nuclear and cytoplasm with bright green and make acidic vesicular organelles (AVOs) with bright red. Images were taken with a fluorescence microscope (magnification, x200). (A) A549 cells were treated with different concentrations of Cur for $48 \mathrm{~h}$. The picture showed that Cur administration increased the amount of AVOs dyed to bright red. (C) A549 cells were pre-treated with 3-MA (2.5 mM) for $3 \mathrm{~h}$, followed by treatment with or without Cur for an additional $48 \mathrm{~h}$. It was shown that 3-methyladenine (3-MA) could suppress autophagy induced by Cur, according to the impaired red fluorescence. (B and D) The percentage of AVOs formation was counted at different treatment groups. (B) Showed that AVOs dyed to bright red were significantly increased in a dose-dependent manner of Cur treatment. Compared with the treatment of Cur alone, the ratio of AVOs in pretreatment of 3-MA group was significantly decreased in (D) indicating the inhibitory effect of 3-MA on Cur-induced autophagy. Three independent experimental images were selected for each treatment concentration and the incomplete cells were removed. Data were represented as mean \pm SEM. $\mathrm{P}<0.05$ was considered to show statistial significance $\left({ }^{* *} \mathrm{P}<0.01\right)$.

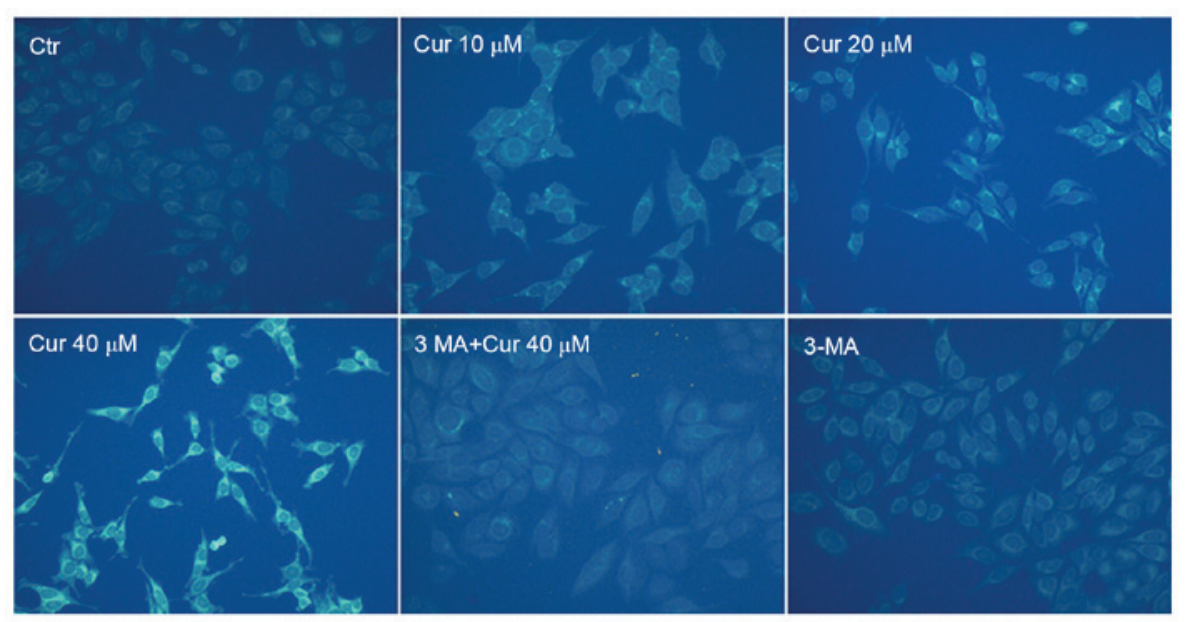

Figure 4. Microscopic detection of autophagic vesicles in A549 cells by MDC staining. The picture showed that curcumin (Cur) administration increased the fluorescence intensity and density which was in a dose-dependent manner of Cur treatment and 3-methyladenine (3-MA) could suppress autophagy induced by Cur, according to the weakened MDC-positive autophagic vacuoles. Images were taken with a fluorescence microscope (magnification, x200). A549 cells were treated with different concentrations of Cur for $48 \mathrm{~h}$ or pre-treated with 3-MA $(2.5 \mathrm{mM})$ for $3 \mathrm{~h}$, followed by treatment with or without Cur for an additional 48 h. Ctr, control.

curcumin treatment, which was at least in part responsible for the reduction in cell viability.

\section{Discussion}

Curcumin having a broad application prospects is considered to be a safe and effective antitumor drug. However the mechanism of antitumor of curcumin is still not fully elucidated. In this study, the human lung adenocarcinoma A549 cell line were selected as experimental cells. We aim to examine the anti-proliferation mechanism of curcumin in A549 cells, especially to confirm autophagy induced by curcumin. Morphologically, curcumin $(40 \mu \mathrm{M})$ administration caused the cells to lose their normal long shuttle shape 

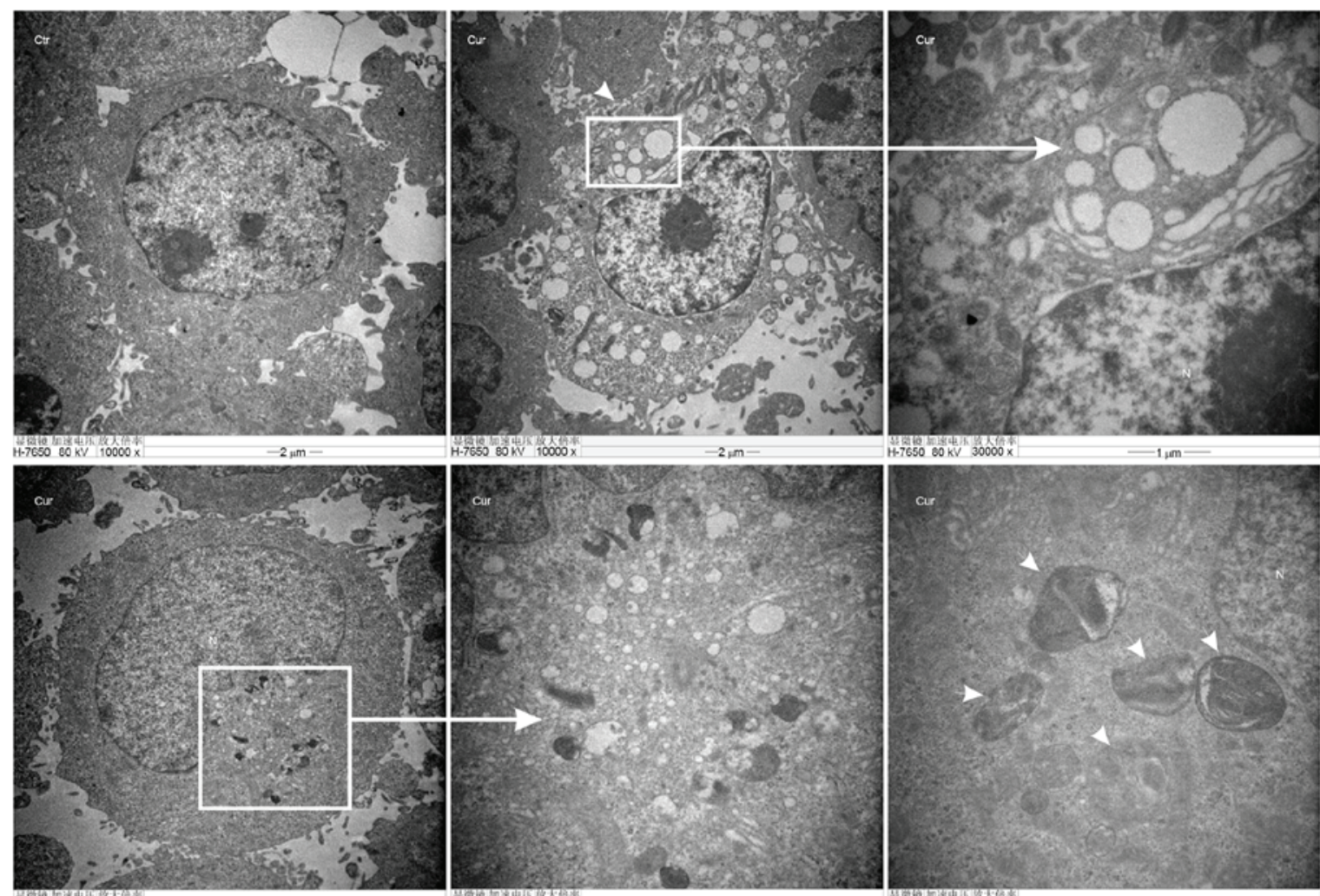

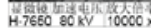

Pr.7.750

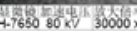

Figure 5. The images of autophagosome were observed by transmission electron microscope in A549 cells. Cells were treated with or without curcumin $(20 \mu \mathrm{M})$ for $48 \mathrm{~h}$. Compared with the control group, a large number of autophagic vacuoles and characteristic autophagosomes (white arrows) were found in the curcumin-treated group. Scale bar, 1 to10 $\mu \mathrm{m}$.

and resulted in the increase of floating suspected dead cells, which appeared to be time dependent (Fig. 1). Curcumin could significantly inhibit the proliferation of human lung adenocarcinoma A549 cells at the concentration and time range we selected (Fig. 2). Previous studies have shown that curcumin suppressed the growth of multiple cancer lines. Here, evidence has been obtained that curcumin, even at very low concentrations, could effectively inhibit the proliferation of lung cancer A549 cells, but the specific mechanism need to be further explored.

More than 3,000 plant species have been reported to treat cancer and inducing apoptotic cell death is plant-derived anticancer drugs mainly mechanism (17). Many natural plant compounds have a shared molecular mechanism for the autophagy function of anti-aging, anticancer and other diseases. Not surprisingly, apoptosis has been recognized as the major toxic mechanism of curcumin in tumor cells $(8,18,19)$. Autophagy may play an important role in tumorigenesis and development, and becomes a new hotspot in tumor research. Abnormal autophagy exists in tumor cells in which autophagic death can play an antitumor effect, which provides a new clue for the treatment of antitumor. Recent studies have shown that autophagy can be activated in tumor cells under the action of some natural botanical ingredients $(20,21)$. These findings highly suggested the importance to further elucidate the relationship between the potential autophagy state and curcumin induced anticancer effect.

Therefore, a variety of methods have been used to verify the relevance of curcumin and autophagy in human lung

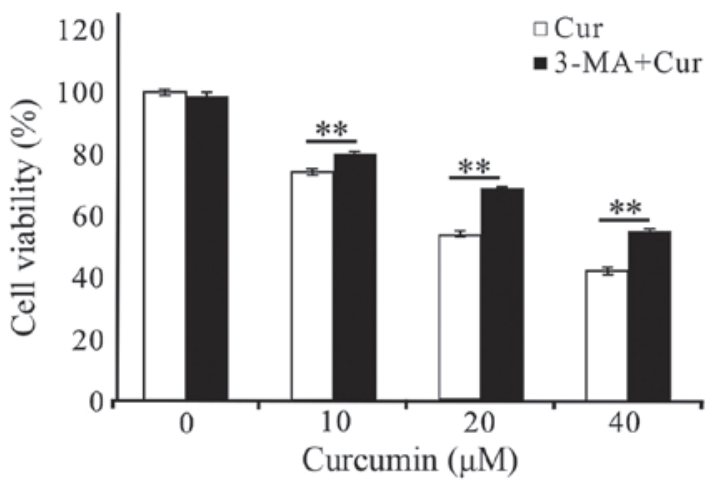

Figure 6. Compared with control [curcumin (Cur) $0 \mu \mathrm{M}$ ] groups pretreatment with 3-methyladenine (3-MA) significantly attenuated the reduction in cell viability at different treatment concentrations of Cur for $48 \mathrm{~h}$ as MTT assay. Data were represented as mean \pm SEM, which are three separate experiments performed in triplicate. $\mathrm{n}=9$ in each group. ${ }^{* *} \mathrm{P}<0.01$. One-way ANOVA followed by LSD multiple comparision t-test.

adenocarcinoma A549 cells here. To elucidate the A549 cell death pathway induced by curcumin, $\mathrm{AO}$ staining and MDC labeling analysises were done to detect the AVOs which suggested a concentration-dependent increase of the fluorescent structures for $48 \mathrm{~h}$ (Figs. 3A and 4). To be more intuitive, the percentage of curcumin-treated cells with AVOs were counted in the pictures, which was consistent with the above results (Fig. 3B). To further confirm the autophagy induced by curcumin in A549, we performed the gold standard for autophagy detection-TEM showing an accumulation of 
double-membrane-enclosed autophagosome in A549 cells after exposure to $20 \mu \mathrm{M}$ of curcumin for $48 \mathrm{~h}$, whereas no autophagosome was observed in untreated cells (Fig. 5). These results collectively indicated that autophagy was induced by curcumin administration in A549 cells in a dose-dependent manner within a certain range. Compared to previous studies (13), we have consistently reached a conclusion that curcumin induced the formation of autophagosome in tumor cells. It is worth mentioning that curcumin could induce autophagy in A549 cells demonstrated in this experiment enriching and contributing to the clinical treatment of lung cancer.

However, retrieving the present studies, it seems still difficult to draw a positive convinciable conclusion that curcumin induced autophagy did exert its anticancer effect on tumor control. On the one hand, nucleotides and free fatty acids promote cell survival (22). On the other hand, autophagy may be capable of ultimately killing cells when allowed to reach its limit (21). Although the dual role of autophagy has not yet reached a consensus, the role in tumor development and treatment can't be ignored. So, what is the role of autophagy in the antitumor effect of curcumin on the proliferation of lung adenocarcinoma A549 cell? This is the focus of our research.

3-MA, used as a typical autophagy inhibitor, interferences the initiation of autophagy by acting on Class III PI3K. The effect of 3-MA on the viability of A549 cells has been examined by MTT assay during the experiment. We found that treatment of A549 cells with 0 and $2.5 \mathrm{mM} \mathrm{3-MA}$ for $48 \mathrm{~h}$ did not affect cell viability, while treatment with 5 and $10 \mathrm{mM} 3-\mathrm{MA}$ caused different degrees of reduction in viability (data not shown). Verifiably, $2.5 \mathrm{mM}$ 3-MA blocked autophagosome formation in cells treated with curcumin according to the results that AVOs stained bright red and MDC fluorescence could be abrogated by pre-treating cells with 3-MA (Figs. 3C and D, and 4). Come together, we selected $2.5 \mathrm{mM}$ of 3 -MA, a safe concentration with a significant autophagic inhibitory effect on A549 cells, which excluding the possible interference effect of 3-MA on A549 cell viability (Fig. 6). What's more, the inhibitory effect of curcumin on A549 cells was attenuated which can be drawn from the significantly increased cell survival rates at each treatment concentrations when pretreated with 3-MA at $2.5 \mathrm{mM}$ (Fig. 6) suggesting that autophagy did take part in the process of curcumin-induced cell death. In the limited exposure time periods and concentration range of curcumin, we support the views of most scholars (23-25) that autophagy is the antitumor mechanism of curcumin rather than a protective mechanism of the A549 cells itself which reminded that the combination of the autophagy inhibitor with curcumin in antitumor therapy requires caution.

Whether inhibition of autophagy has an effect on cell apoptosis, we do not know. Recent studies have pointed towards a closely interplay between autophagy and apoptosis involved in the process of cell death (26-28). So, we apt to think that apoptosis and autophagy share some common signaling pathways and are mutually regulated. For our results, a large number of autophagic vacuoles and characteristic autophagosomes were found in the curcumin-treated group compared with the control group under TEM. We have reason to suspect that prolonged inhibition of autophagy stimulates apoptosis. Considering there is a complex interaction between curcumin-induced apoptosis and autophagy of A549 cells, which contributes to curcumin inhibiting cell survival, further validation at the protein and gene levels should be undertaken.

In conclusion, evidences have been gained that curcumin could significantly inhibit the proliferation of A549 cells in this study, while autophagy was gradually taking in charge as the concentration of curcumin increased. Due to the wide range of effects of natural compounds and the complexity of procedural death we can only draw conclusions that the promotion of tumor cell autophagy activity induced by curcumin even induced autophagic death is a potential tumor treatment within a limited range. The effects of curcumin may differ depending on cell types. There may be having another role in anticancer effects on other exposuring concentrations or treat-times of curcumin, wishing to be in-depth studied later. The study of non-apoptotic cell death mechanisms induced by curcumin would provide targets of plant-derived compounds for future cancer therapeutics and become the new direction of antitumor researches.

\section{Acknowledgements}

The project was supported by the National Natural Science Foundation of China (grant no. 81172598).

\section{References}

1. Jemal A, Bray F, Center MM, Ferlay J, Ward E and Forman D: Global cancer statistics. CA Cancer J Clin 61: 69-90, 2011.

2. Gupta SC, Kismali G and Aggarwal BB: Curcumin, a component of turmeric: From farm to pharmacy. Biofactors 39: 2-13, 2013.

3. Gupta SC, Patchva S and Aggarwal BB: Therapeutic roles of curcumin: Lessons learned from clinical trials. AAPS J 15: 195-218, 2013.

4. Liu D, You M, Xu Y, Li F, Zhang D, Li X and Hou Y: Inhibition of curcumin on myeloid-derived suppressor cells is requisite for controlling lung cancer. Int Immunopharmacol 39: 265-272, 2016.

5. Watson JL, Greenshields A, Hill R, Hilchie A, Lee PW, Giacomantonio CA and Hoskin DW: Curcumin-induced apoptosis in ovarian carcinoma cells is p53-independent and involves p38 mitogen-activated protein kinase activation and downregulation of Bcl-2 and survivin expression and Akt signaling. Mol Carcinog 49: 13-24, 2010.

6. Chang CC, Fu CF, Yang WT, Chen TY and Hsu YC: The cellular uptake and cytotoxic effect of curcuminoids on breast cancer cells. Taiwan J Obstet Gynecol 51: 368-374, 2012.

7. Kang JH, Kang HS, Kim IK, Lee HY, Ha JH, Yeo CD, Kang HH, Moon HS and Lee SH: Curcumin sensitizes human lung cancer cells to apoptosis and metastasis synergistically combined with carboplatin. Exp Biol Med (Maywood) 240: 1416-1425, 2015.

8. Han X, Deng S, Wang N, Liu Y and Yang X: Inhibitor effects and molecular mechanisms of tetraphydrocurcumin against human breast cancer MCF-7 cell. Food Nutr Res 60: 30616, 2016.

9. Xia YQ, Wei XY, Li WL, Kanchana K, Xu CC, Chen DH, Chou PH, Jin R, Wu JZ and Liang G: Curcumin analogue A501 induces G2/M arrest and apoptosis in non-small cell lung cancer cells. Asian Pac J Cancer Prev 15: 6893-6898, 2014.

10. Shinojima N, Yokoyama T, Kondo Y and Kondo S: Roles of the Akt/mTOR/p70S6K and ERK1/2 signaling pathways in curcumin-induced autophagy. Autophagy 3: 635-637, 2007.

11. Guan F, Ding Y, Zhang Y, Zhou Y, Li M and Wang C: Curcumin suppresses proliferation and migration of MDA-MB-231 breast cancer cells through autophagy-dependent Akt degradation. PLoS One 11: e0146553, 2016.

12. Xiao K, Jiang J, Guan C, Dong C, Wang G, Bai L, Sun J, Hu C and Bai C: Curcumin induces autophagy via activating the AMPK signaling pathway in lung adenocarcinoma cells. J Pharmacol Sci 123: 102-109, 2013. 
13. Thongrakard V, Titone R, Follo C, Morani F, Suksamrarn A, Tencomnao T and Isidoro C: Turmeric toxicity in A431 epidermoid cancer cells associates with autophagy degradation of anti-apoptotic and anti-autophagic p53 mutant. Phytother Res 28: 1761-1769, 2014.

14. White E: Deconvoluting the context-dependent role for autophagy in cancer. Nat Rev Cancer 12: 401-410, 2012.

15. Zhou GZ, Sun GC and Zhang SN: The interplay between autophagy and apoptosis induced by one synthetic curcumin derivative hydrazinobenzoylcurcumin in A549 lung cancer cells. J Biochem Mol Toxicol 29: 267-273, 2015.

16. Yu W, Tao Z, Hanyan S and Bei H: Study on the relationship between autophagy and apoptosis in A549 cells induced by curcumin analogue EF24. Chin J Cell Biol 6: 590-596, 2012.

17. Gali-Muhtasib H, Hmadi R, Kareh M, Tohme R and Darwiche N: Cell death mechanisms of plant-derived anticancer drugs: Beyond apoptosis. Apoptosis 20: 1531-1562, 2015.

18. Montazeri M, Sadeghizadeh M, Pilehvar-Soltanahmadi Y, Zarghami F, Khodi S, Mohaghegh M, Sadeghzadeh H and Zarghami N: Dendrosomal curcumin nanoformulation modulate apoptosis-related genes and protein expression in hepatocarcinoma cell lines. Int J Pharm 509: 244-254, 2016.

19. Guo S, Long M, Li X, Zhu S, Zhang M and Yang Z: Curcumin activates autophagy and attenuates oxidative damage in EA.hy926 cells via the Akt/mTOR pathway. Mol Med Rep 13: 2187-2193, 2016

20. Rigacci S, Miceli C, Nediani C, Berti A, Cascella R, Pantano D, Nardiello P, Luccarini I, Casamenti F and Stefani M: Oleuropein aglycone induces autophagy via the AMPK/mTOR signalling pathway: A mechanistic insight. Oncotarget 6: 35344-35357, 2015

21. Selvaraj S, Sun Y, Sukumaran P and Singh BB: Resveratrol activates autophagic cell death in prostate cancer cells via downregulation of STIM1 and the mTOR pathway. Mol Carcinog 55: 818-831, 2016

22. Jaroonwitchawan T, Chaicharoenaudomrung N, Namkaew $J$ and Noisa P: Curcumin attenuates paraquat-induced cell death in human neuroblastoma cells through modulating oxidative stress and autophagy. Neurosci Lett 636: 40-47, 2017.
23. Wang K, Zhang C, Bao J, Jia X, Liang Y, Wang X, Chen M, Su H, Li P, Wan JB and He C: Synergistic chemopreventive effects of curcumin and berberine on human breast cancer cells through induction of apoptosis and autophagic cell death. Sci Rep 6: 26064, 2016

24. Zanotto-Filho A, Braganhol E, Klafke K, Figueiró F, Terra SR, Paludo FJ, Morrone M, Bristot IJ, Battastini AM, Forcelini CM, et al: Autophagy inhibition improves the efficacy of curcumin/temozolomide combination therapy in glioblastomas. Cancer Lett 358: 220-231, 2015.

25. Papandreou I, Verras M, McNeil B, Koong AC and Denko NC: Plant stilbenes induce endoplasmic reticulum stress and their anti-cancer activity can be enhanced by inhibitors of autophagy. Exp Cell Res 339: 147-153, 2015.

26. Jing K, Song KS, Shin S, Kim N, Jeong S, Oh HR, Park JH, Seo KS, Heo JY, Han J, et al: Docosahexaenoic acid induces autophagy through $\mathrm{p} 53 / \mathrm{AMPK} / \mathrm{mTOR}$ signaling and promotes apoptosis in human cancer cells harboring wild-type p53. Autophagy 7: 1348-1358, 2011.

27. Kumar D, Das B, Sen R, Kundu P, Manna A, Sarkar A, Chowdhury C, Chatterjee M and Das P: Andrographolide analogue induces apoptosis and autophagy mediated cell death in U937 cells by inhibition of PI3K/Akt/mTOR pathway. PLoS One 10: e0139657, 2015

28. Ge J, Liu Y, Li Q, Guo X, Gu L, Ma ZG and Zhu YP: Resveratrol induces apoptosis and autophagy in T-cell acute lymphoblastic leukemia cells by inhibiting Akt/mTOR and activating p38-MAPK. Biomed Environ Sci 26: 902-911, 2013. 